\title{
L'IMAGE DU MONTENEGRO ET UN POINT DU VUE SUR LA NARRATION DANS LETTRES SUR L'ADRIATIQUE ET LE MONTENEGRO DE XAVIER MARMIER
}

Marija Dulović, Université de Strasbourg, mddulovic@gmail.com

$10.31902 /$ fll.29.2019.14
UDK 821.133.1.09-922"17"

Résumé : Nous nous proposons de présenter Lettres sur l'Adriatique et voyage au Monténégro de Xavier Marmier, un voyageur et écrivain français qui a fait beaucoup de voyages autour du monde, y compris le Monténégro en 1852. Son trajet est décrit en deux volumes, dont le second est majoritairement consacré au Monténégro (six sur huit chapitres). Il est resté au Monténégro pendant deux mois et a écrit un beau témoignage de cette période, en s'appuyant à la fois aux faits historiques, aux témoignages des habitants et à ses impressions personnelles. Dans la première partie de notre étude, nous présentons l'auteur et son œuvre, et fournissons ensuite une sorte de synthèse de son écriture sur le Monténégro, en nous concentrant sur les points tels que le contexte historique, les conditions de vie du peuple ainsi que ses mœurs et traditions. Dans la deuxième partie, nous proposons une réflexion sur la narration et plus précisément sur la temporalité narrative dans cette œuvre. Nous cherchons à identifier les différents repères temporels afin d'expliquer l'emploi de certains temps verbaux dans le récit.

Mots clés : Monténégro, Xavier Marmier, récit de voyage, narration, temporalité.

\section{Introduction}

En vue d'aborder le sujet de la mémoire sous l'angle comparatif francomonténégrin, nous nous proposons de présenter un récit de voyage français portant sur le Monténégro, le livre « Lettres sur l'Adriatique et voyage au Monténégro » que Xavier Marmier a écrit pendant son séjour au Monténégro en 1852 et publié en 1854 . Nous commencerons par une brève présentation de l'auteur, retracerons ensuite quelques extraits pertinents du livre, dans le but de décrire le Monténégro et les conditions de vie de l'époque. Ce qui nous amènera à proposer enfin une courte étude portant sur la question de la mémoire de point de vue temporel, à savoir sur l'emploi des temps verbaux dans ce type d'écriture.

\section{Xavier Marmier}

Marmier (1808-1892) était membre de l'Académie française, écrivain, voyageur et traducteur. II s'agit effectivement d'un écrivain dont les œuvres 
sont très variés : nombreuses histoires, lettres, essais, romans, traductions de pièces de théâtre et de contes, poésies, et enfin récits de voyages. L'intérêt pour les récits de voyages s'inscrit dans la tendance générale de son époque ; en effet, « dans les années 1830 le goût du voyage (et de la littérature de voyage) envahit l'Europe » (Piroska 2001, 217).

Étant un voyageur passionné, Marmier a commencé à noter ses impressions concernant les pays qu'il était en train de visiter. Nous apprenons ainsi, dans la préface de son journal, qu' « il a couru de Stockholm à Jérusalem, du Rhin au Nil ; aussi peut-on dire sans exagération que Marmier a parcouru une notable portion du globe » (Marmier 1968, 39).

Nous pouvons supposer aussi que le propos que Marmier « fut le touriste idéal, et même plus qu'un touriste " (Marmier 1968, 19) s'explique par le fait qu'il se préparait consciencieusement pour chaque voyage, et ne partait pas sans s'être informé sur des faits historiques, géographiques et littéraires sur le pays concerné. Et ce qui est le plus important, il parlait plusieurs langues étrangères : I'allemand, italien, espagnol, anglais, danois, suédois, hollandais et russe. Nous pouvons ainsi en déduire qu'il se sentait bien à l'aise partout où il se rendait, comme ces écritures en témoignent. Par ailleurs, nous savons qu'il " possédait deux qualités indispensables à un bon voyageur : la gaieté et la bienveillance " (Marmier 1968, 20) et qu'il était parfaitement capable de s'accommoder « de tout, des rigueurs du climat, des moyens de transport rudimentaires, de l'alimentation peu ragoûtante " (Marmier 1968, 20).

II a publié plusieurs œuvres retraçant ces voyages, parmi lesquels ils convient de mentionner Lettres sur I'Islande (1837), Histoire de la littérature en Danemark et en Suède (1839), Du Rhin au Nil (1847), Lettres sur l'Algérie (1847), Lettres sur la Russie, la Finlande et la Pologne (1848), Lettres sur l'Amérique (1852), Lettres sur l'Adriatique et le Monténégro (1854), etc. La valeur de ces écritures est sans doute inestimable, car elles représentent un témoignage historique très fidèle sur les pays différents.

\section{Voyage au Monténégro}

En ce qui concerne son voyage au Monténégro, à l'époque le petit pays inconnu, Marmier l'a fait en 1852. Nous apprenons dans son journal qu'il avait décidé d'entreprendre ce voyage dans le but de comparer les Slaves du nord (qu'il a rencontrés en Russie) à ceux du sud, et d'étudier l'histoire, les mœurs et les traditions de ces derniers. II est parti de Strasbourg, par l'Allemagne et l'Italie, et a pris un bateau de Trieste pour Zara en Dalmatie. II s'est ensuite rendu à Split, Dubrovnik, Risan et Kotor, et a puis voyagé de Kotor à Cetinje à cheval. Au cours de son séjour d'à peu près trois mois, Marmier a recueilli les différents témoignages, renseignements, faits historiques et culturels, aussi bien que ses propres observations et impressions, qu'il a ensuite publié dans deux volumes de « Lettres sur l'Adriatique et le Monténégro ». 
Six chapitres du deuxième tome de ce livre retracent les impressions sur le Monténégro et sont intitulés comme suit :

III Les Bouches de Cattaro ${ }^{1}$

IV Njeguši

V Histoire du Monténégro

VI Statistique. Administration

VII Mœurs et coutumes

VIII Les chants serbes.

Afin d'esquisser le contexte général de l'époque, il nous semble opportun de dire que le voyage a eu lieu en 1852, l'époque où le Monténégro était gouverné par le prince Danilo Petrović Njegoš, successeur du grand Petar II Petrović Njegoš. Le pays, bien entendu, était sous règne de l'empire ottoman.

Comme nous l'avons suggéré plus haut, Marmier est d'abord arrivé à Risan et sa première impression concernant le Monténégro porte sur son nom. Souhaitant expliquer le nom du pays, signifiant la Montagne Noire, l'auteur trouve que ce nom est tout à fait justifié en insistant sur l'apparence noir du pays et en employant l'adjectif noir plusieurs fois dans un seul extrait :

" J'ai traversé cette baie ${ }^{2}$ en un jour d'orage. Elle me rappelait par son étonnant aspect les plus sombres peintures de Byron. (...) Mais le ciel était noir ; des masses de nuages noirs flottaient autour de nous, et si nous n'avions su que les hautes crêtes au pied desquelles nous naviguions portaient depuis longtemps le nom de Tzerna Gora, de Monténégro, de montagne Noire, nous n'aurions pu leur donner une plus juste dénomination » (Marmier 1854, 89-90).

Il dira ensuite :

" quiconque aura vu le front calcaire, la face grise des cimes du Monténégro sous les sombres nuées qui les enveloppent en un jour d'orage, comprendra aisément qu'on leur ait donné le nom de Montagne Noire... " (Ibidem, 147).

II s'aperçoit en plus que le Monténégro est très riche en pierres, ce qui s'explique par la légende que les gens du pays lui ont racontée. La légende dit que Dieu, ayant terminé de créer le globe terrestre, s'en allait un jour avec un sac plein de pierres qu'il voulait semer çà et là. Malheureusement, quand il passait par le Monténégro, son sac s'est déchiré. L’auteur ainsi conclut :

${ }^{1}$ Kotor.

${ }^{2}$ Boka. 
« Le sac était d'une rare dimension, le trou qui s'y fit était large, et par là tombèrent les amas de pierres qui couvrent la surface de cette contrée. De tous côtés, nous ne voyons que des masses de rocs... " (Ibidem, 165).

Marmier est tout de même impressionné par la beauté du pays, qu'il décrit comme " œuvre solennelle de Dieu » (Ibidem, 94) en s'exclamant avec admiration " quels délicieux points de vue, et quelle variété de tableaux, de tout côté » (Ibidem, 93).

L'auteur nous dévoile également les différentes caractéristiques des gens. Il retrace l'apparence physique des habitants et remarque bien que chaque village a son propre costume. C'est pour cette raison qu' " on peut dire au simple aspect du vêtement : cet homme vient de Castelnuovo ${ }^{3}$, cette jeune fille de Dobrota, cette femme de Perasto ${ }^{4}$ " (Ibidem, 99). II souligne ensuite comme un fait curieux la force vocale des Monténégrins en constatant qu'ils parlent généralement de voix haute et poussent souvent des cris, comme signe d'appel à quelqu'un dans la proximité. II observe également que les Monténégrins sont très braves, et, même si peu nombreux, ils s'engagent souvent dans les luttes contre les Turcs, ou les uns contre les autres en raison de la loi de la vengeance, qui est toujours si présente et « si profondément enracinée dans l'esprit de ces habitants des montagnes " (Ibidem, 135) vu que " chaque Monténégrin est, dès sa jeunesse, résolu (...) à se faire le médecin de son honneur » (Ibidem, 140).

Les Monténégrins s'impliquent souvent dans les combats contre les Turcs " par leur animosité chrétienne, par leur haine héréditaire » (Ibidem, 179). Il y a même une coutume extraordinaire : pour chaque tête de Turc qu'un Monténégrin ramène à Cetinje, il reçoit une prime, comme, Marmier l'observe, les paysans de France pour une tête de loup (Ibidem, 179). II remarque également que, même si les gens ne sont pas formellement éduqués, tout petit garçon écoute raconter

" les luttes glorieuses de sa tribu, les batailles dans lesquelles sa famille elle-même s'est signalée. C'est là son éducation, c'est là sa poésie et ses chroniques. C'est là le mobile de sa pensée et la première conception de sa destinée » (Ibidem, 306).

En considération des conflits fréquents avec les Turcs, l'auteur nous signale que les Monténégrins sont habituellement bien armés et le poignard est devenu la partie intégrante de l'habit de chaque homme. II remarque de même qu'à Njeguši quelquefois, quand on porte un enfant à l'église pour le faire baptiser " on place dans ses langes un handjar et un pistolet, afin qu'il aille, avec ce signe du soldat, recevoir son signe de chrétien " (Ibidem, 160). Marmier constate ensuite que les Monténégrins sont fort religieux. La communauté

\footnotetext{
${ }^{3}$ Herceg Novi.

${ }^{4}$ Perast.
} 
chrétienne grecque y est la plus nombreuse, mais Marmier nous fait remarquer cependant qu'un grand nombre d'

" ecclésiastiques grecs qu'on voit passer majestueusement avec une belle robe en soie, une large ceinture, une barbe vénérable et de longs cheveux noirs bouclés, ne savent pas même lire. Ils apprennent seulement à célébrer les cérémonies de l'Église, à réciter par cœur les passages usuels de la liturgie, et d'ordinaire sont remplacés par leurs fils, à qui ils donnent les mêmes leçons » (Ibidem, 98).

II s'agit effectivement d'un fait bien connu et une tendance régulière de cette période ; nous savons que Njegoš a aussi décrit dans « Gorski vijenac » ${ }^{5}$ le caractère de pope Mićo, qui ne savait pas lire mais avait seulement appris par cœur les passages de la Bible et différentes cérémonies de l'église.

L'auteur décrit également l'habitation typique de cette époque. Les Monténégrins habitent dans des petites maisons en pierre, qui sont habituellement constituées d'une seule pièce, rarement deux. Au milieu de cette pièce se trouve le foyer, et c'est là « qu'on se chauffe et qu'on fait cuire les aliments » (Ibidem, 291). Le mobilier est très modeste.

Parmi les différentes coutumes décrites, il convient de faire mention de la cérémonie de mariage. Nous apprenons ainsi que les mariages sont normalement arrangés ; c'est le père d'un garçon qui choisit la fille pour son fils. II rend ensuite visite au père de la fille pour lui faire sa demande et en lui offrant un verre de vin. "Si le vin est accepté, l'union est résolue " (Ibidem, 292). Même si les gens vivent en général dans la pauvreté, ils tiennent beaucoup à la célébration des mariages. Toute famille fait des économies et garde des provisions pour une telle occasion, qui est normalement organisée en luxe ; on y sert des meilleurs plats et boissons. La cérémonie est parfois célébrée pendant huit jours

« en grande pompe, par une quantité de détonations de coups de pistolet et de coups de fusil. (...) Puis la famille, qui dans cette circonstance solennelle a tué plusieurs moutons et vidé plusieurs tonnelets de vin, rentre dans la sévérité de son régime accoutumé : des galettes de maïs cuites sous la cendre, des pommes de terre bouillies, de temps à autre un peu de viande fumée, tel est le menu de la semaine, et la loi des jours de carême, si nombreux dans la religion grecque, est fidèlement observée (Ibidem, 292-293).

Marmier consacre beaucoup d'espace à la situation des femmes ; il observe que la jeune mariée est " reine un jour, esclave ensuite ", suggérant qu'elle est traitée avec tout le respect qui lui est dû un seul jour de sa vie : celui

\footnotetext{
${ }^{5}$ La couronne des montagnes.
} 
de son mariage. La femme monténégrine vit effectivement, dès sa naissance, dans les conditions peu enviables, et l'auteur affirme :

« Les pauvres femmes ! À quel état d'humilité et de servitude elles sont astreintes ! (...) L’homme est leur maître impérieux et fier, dédaigneux et cruel. L'homme leur fait porter, comme à ses bêtes de somme (...) les plus lourds fardeaux ; et, lorsque, chemin faisant, elles rencontrent un homme de leur connaissance, elles n'oseront point le saluer familièrement : elles iront avec respect s'incliner devant lui comme devant leur seigneur et lui baiser la main » (Ibidem, 141).

Faisant en outre allusion à la malédiction d'Adam et Ève :

« Dieu avait dit à la femme : Tu enfanteras dans les douleurs; et à I'homme : Tu mangeras ton pain à la sueur de ton front. »

Marmier observe qu'au Monténégro la femme « joint aux douleurs de l'enfantement les sueurs du travail de l'homme " (Ibidem, 142). Contrairement à la position difficile de la femme, le mari est désigné comme le souverain-maître ( $/ b i$ dem, 169) :

" Le gouvernement de la maison est tout entier laissé au père de la famille. On l'écoute avec respect, on accepte ses ordres avec soumission, on l'appelle le Gospodar » (Ibidem, 276).

Malgré ce fait, ce sont les femmes « sur lesquelles pèse, on peut le dire, tout le poids de l'existence " (Ibidem, 297), ce sont les " ouvrières par excellence » (Ibidem, 293). En raison des conflits courants avec les Turcs, les hommes sont souvent absents ; c'est donc la femme qui tient ménage, s'occupe des enfants et de toutes les autres tâches ménagères. La force morale et le courage exceptionnel de ces femmes se voient dans leur détermination pour aider et leur volonté de faire des sacrifices. L'auteur signale

«Dans les occasions graves, on les a vues quitter intrépidement leur foyer pour s'associer aux dangers de leurs frères, de leurs maris. Si elles ne combattaient pas avec le fusil ou le handjar, elles aidaient du moins au combat. Dans le voyage que je viens de faire, j'en ai rencontré plusieurs qui, au péril de leur vie, portaient des sacs de cartouches et des sacs de balles aux soldats de leur communauté, luttant contre les Turcs sous les murs de Žabljak » (Ibidem, 300). 
Finalement, bien que ces " braves femmes de la montagne Noire " (Ibidem, 298) soient traitées en inférieur, elles sont très respectées, et Marmier indique que " chacune d'elles peut voyager sans crainte de nuit comme de jour par les routes les plus désertes du Monténégro. Personne n'osera leur faire la moindre injure " (Ibidem, 297). Elles sont vêtues de façon la plus misérable et sont même parfois décrites comme sales, mais sont toutefois très belles, et l'auteur indique que les Monténégrins sont fort jaloux de leurs femmes, et qu'on " ne pourrait, sans s'exposer à une vengeance mortelle, se hasarder à faire à I'une de ces sauvages beautés une galante déclaration » (Ibidem, 140).

\section{Temporalité}

Dans cette deuxième partie de notre communication, nous cherchons à aborder le sujet de mémoire sous l'angle de la narration, ou plus précisément de la temporalité narrative et l'emploi des temps verbaux dans ce récit. Nous nous intéressons ainsi à identifier les différents temps verbaux et leurs repères, aussi bien que leurs rôles dans les contextes variés. Deux temps s'y distinguent en particulier : le présent et le passé simple de l'indicatif.

Signalons d'abord que le récit est majoritairement raconté à la première personne. Il s'agit des parties appartenant à la " focalisation interne " (Genette 1972,209 ) où le narrateur est en même temps l'acteur du récit. II raconte son histoire en temps réel vu qu'il décrit ce qu'il est en train de vivre ; son récit se présente à peu près comme son journal intime. D'autres parties sont relatées à la focalisation externe, où le narrateur raconte les événements non comme leur participant direct mais comme leur témoin ; dans le présent cas il narre les faits qu'il a précédemment appris.

L'histoire est majoritairement relatée au présent, ce qui s'explique par le simple fait que le narrateur expose les faits comme leur témoin direct : il raconte ce qu'il est en train de voir/vivre. Les faits exposés sont conséquemment approchés aux lecteurs. Cette écriture est en quelque sorte le reportage direct, qui se réfère apparemment à la réalité immédiate du narrateur. Nous pouvons présumer ainsi que Marmier notait au fur et à mesure ses impressions. Ceci s'illustre par l'exemple suivant :

" Donc nous voici dans un village monténégrin, c'est-à-dire dans un cordon d'habitations éparses, adossées à un rocher ou égrenées le long d'un coteau, et nous nous arrêtons au hasard devant la première qui se présente à nous. Nul architecte $n$ 'en a dessiné la façade, et le maçon qui l'a construite ne s'est pas inquiété de la rectitude de ses angles. Des pierres posées l'un sur l'autre, tant bien que mal, jusqu'à une hauteur de huit à dix pieds, en forment les quatre murs. Un toit en chaume la recouvre. Le toit en tuile est une exception » (Ibidem, 200). 
Le narrateur fait le récit « en direct ». La préposition voici sert à indiquer la présence du sujet (nous, en l'occurrence) dans un lieu spécifique (un village monténégrin) à un moment donné (le présent du narrateur). La présence dans le village est donc actuelle au moment où l'on décrit dans le livre. La suite de l'extrait est également repérée au présent : nous nous arrêtons, se présente, forment, recouvre, est exception. Inutile de le dire, le présent est utilisé ici pour marquer les procès ayant lieu presque simultanément avec le procès d'écriture. Trois procès au milieu sont repérés au passé composé, indiquant évidemment les procès passés, donc antérieurs à ceux au présent. Comme nombreuses grammaires le soulignent, le passé composé est utilisé pour repérer les événements en lien avec le présent du narrateur.

Observons d'autres passages narratifs faits exclusivement au présent :

« En parcourant d'une de ses extrémités à l'autre les Bouches de Cattaro, tantôt il semble qu'on glisse sur les flots d'une paisible rivière, tantôt sur un des lacs de Suisse. Autour de leurs différents bassins s'élèvent des montagnes gigantesques, dont nulle plante ne couronne la tête chauve, mais sur leurs flancs verdoient sans cesse des bois d'oliviers, de figuiers, d'amandiers ; à leur base s'épanouissent des jardins fleuris et dans toute la longueur du canal, sur ses deux bords, comme sur ceux du Bosphore, se déroule un collier de riants villages, de jolies maisons entourées de fécondes plantations » (Ibidem, 90-91).

Le présent employé ici, tout comme dans le premier exemple, a une valeur descriptive : le narrateur donne une vision subjective de son entourage. Nous pouvons facilement imaginer que l'auteur, qui est en train d'observer l'habitation d'un village (exemple 1) ou de passer par la Baie de Kotor (exemple 2), note ses impressions, notamment au présent.

Par la suite, nous retrouvons aussi le présent narratif (ou historique). Les narrateurs employant ce type de présent « become so involved in their stories that they recount the action as if they were reliving it simultaneously with its telling, or, alternatively, that they experience the events subjectively rather than viewing them objectively distanced in the past " (Fleichmann 1990, 75). Ce présent figure dans le texte souvent en combinaison avec le passé composé, qui est repéré par rapport à ce présent. Par exemple, parlant des mariniers de Boka, on dit :

"Quiconque a parmi eux gravi heureusement ces divers échelons, veut se reposer de sa carrière au lieu d'où il est parti et rapporter sa couronne de florins à son foyer. II revient donc à Dobrota, emploie une partie de sa fortune à élargir, à embellir la maison que son père lui a léguée, ou en construit une nouvelle. Là, il dépose avec orgueil tout ce qu'il a re- 
cueilli dans ses voyages : objets de luxe, meubles étrangers. Puis il veut avoir son jardin, son enclos, et il paye à un prix énorme une parcelle de terre... » (Ibidem, 102-103).

En employant le présent ici, le narrateur insiste sur l'accomplissement de chaque événement, mais crée aussi l'effet de répétition, qui indique qu'il ne s'agit pas d'un cas isolé parmi les mariniers mais d'un comportement largement accepté parmi eux. Même si le présent narratif évoque en général " des événements passés, réels ou fictifs » (Riegel, Pellat \& Rioul 2013, 533) et " raconte au présent une histoire passée » (Leeman-Bioux 1994, 149), nous pouvons présumer que les procès décrits dans l'exemple cité ne sont pas repérés par rapport à un moment passé spécifique (ni par rapport au moment présent), mais sont valables à tout moment. D'où la détermination du présent comme une " forme neutre » (Barceló \& Bres 2006, 124), ce qui veut dire qu'il peut situer les événements à toute époque temporelle : passée, présente ou future. Le passé composé, pour sa part, est utilisé pour marquer les événements antérieurs au présent actuel des mariniers (la période après leur voyage) ; ce sont les procès qui se sont produits avant leur rentrée à Dobrota.

Le présent est souvent associé aux formes passées : au passé composé, comme nous venons de le voir, ou même au passé simple. La corrélation présent/passé simple se rencontre dans les contextes où il y a un décrochage au niveau de distanciation par rapport aux événements rapportés. Ce sont les cas où le narrateur prend de recul par rapport à son actualité présente pour relater une histoire passée ou pour renvoyer à un souvenir particulier :

« J'arriverai bientôt à Cattaro; je dois commencer par dire ce qu'on voit sur le canal avant de parvenir à cette curieuse petite ville. Castelnuovo fut fondée à la fin du XIVe siècle par Tvarko, roi de Servie, et tomba ensuite au pouvoir des Turcs. En 1538, les Vénitiens unis aux Espagnols assiégèrent cette ville, s'en emparèrent et y construisirent un fort qui subsiste encore et porte le nom de Spagnuolo » (Ibidem, 95-96).

Le narrateur emploie tout d'abord le futur simple et le présent. Ces deux temps font partie de son actualité, et sont donc repérés au moment présent, celui de l'écriture. Nous observons tout de suite une transition vers le passé simple, que le narrateur utilise pour raconter l'histoire au sujet de la fondation de Herceg Novi. Il s'agit, bien évidemment, d'une histoire qui n'appartient pas à l'actualité du narrateur mais est complètement découpée du moment présent. C'est pour cette raison qu'il opte pour le passé simple et non pour le passé composé. Contrairement au passé composé, le passé simple « n'est pas formellement mis en relation avec le moment de l'énonciation " et est donc " plus apte à rapporter des faits passés coupés du présent de l'énonciateur, ce qui leur confère une grande autonomie ॥ (Riegel, Pellat $\boldsymbol{\&}$ Rioul 2013, 538). Après la série au passé 
simple, le narrateur revient au présent : ils y construisirent un fort qui subsiste encore et porte le nom de Spagnuolo. La transition du passé simple (l'énonciation historique en termes de Benveniste 1966) au présent (l'énonciation de discours) dans une même phrase peut paraître inattendue, mais elle se justifie par le simple fait que le fort se maintient encore au moment où le narrateur en parle.

L'introduction d'un passage au passé simple instaure certainement une distance énonciative par rapport au sujet du récit :

"Au milieu du limpide espace qui s'ouvre en face de ce village apparaissent deux petites îles charmantes. L'une s'appelle l'île Saint-Georges ; l'autre l'île de la Madone du Scapulaire. Sur la première est un couvent grec ; sur l'autre une chapelle catholique, vénérée dans tout le pays. Une tradition rapporte qu'au XV siècle, des marins virent un soir luire sur cette île, alors déserte, une clarté extraordinaire. Les plus hardis, désirant savoir la cause de ce phénomène, mirent leur chaloupe à l'eau et trouvèrent sur une pointe de roc une image de la Vierge qui avait été déposée là par une main inconnue. Ils la prirent et l'emportèrent respectueusement dans l'église de Perasto » (Ibidem, 92).

" Lorsqu'il fut appelé à succéder à son oncle, Pierre II avait à peine dixhuit ans, et n'avait pas encore pris un des ordres minimes de la prêtrise. Un évêque serbe le fit diacre, et en 1833, il alla à Saint-Pétersbourg recevoir du saint synode sa dignité épiscopale. À son retour, il commença son règne, et qui dit le règne d'un prince monténégrin, dit une existence fort peu paisible. Pierre eut à lutter contre les Turcs... » (Ibidem, 256-257).

Le premier extrait commence au présent descriptif (s'ouvre, apparaissent, s'appelle, est un couvent). Le dernier présent de l'extrait rapporte introduit une suite au passé simple portant sur l'histoire de la tradition, effectivement coupée du moment présent. Dans le deuxième exemple cité, nous observons également l'emploi du passé simple repérant les événements du passé lointain. Nous pouvons constater que, lorsque le narrateur veut introduire un événement écarté du présent du récit (à savoir la visite du Monténégro), il emploie le passé simple. Ce dernier n'est pas repéré par rapport au moment présent mais à un autre repère temporel défini dans le texte (dans les cas cités ici, les années 1538 et 1833, XV' siècle). 


\section{Conclusion}

Nous pouvons conclure tout d'abord que Marmier interprétait dans ce livre à la fois les rôles de touriste, d'écrivain et d'historien. Il visait à présenter la société, l'histoire et les caractéristiques géographiques du Monténégro, aussi bien que son administration, les mœurs et les traditions des habitants. Tout cela est englobé dans l'ensemble de ses impressions personnelles qu'il a partagées soigneusement et d'une manière littéraire. Il s'est appliqué à nous raconter I'histoire du pays et à décrire la vie du peuple de façon très objective et réelle. "Au début du XIX siècle, les écrivains de récits de voyage visent à transmettre des idées au public, mais ils privilégient la représentation de l'image objective du monde, mettant le lecteur en présence de pays et de paysages familiers. (...) L'écrivain garde une certaine distance et ne laisse apparaître que fort peu sa propre subjectivité " (Piroska 2001, 217). Il s'agit de ce fait d'un témoignage fidèle et précieux du Monténégro de l'époque. Pour les buts de la présente étude, nous n'avons présenté que les parties du livre qui nous ont semblé les plus pertinents, mais il s'agit sans doute d'une œuvre digne d'intérêt qui mérite de retenir l'attention de tout lecteur.

Nous avons ensuite vu que ce genre d'écriture est assez intéressant au niveau textuel et en termes de l'emploi des temps verbaux, ce qui nous a permis d'effectuer une analyse intéressante. Nous avons pu observer que le présent est employé de façon prédominante, vu que l'auteur rapportait ses impressions comme dans un reportage direct. Le présent est toutefois souvent accompagné des temps passés, tels que le passé composé et le passé simple. Le passé composé est utilisé pour repérer un événement passé, lorsque le narrateur établit un lien avec le moment présent. En revanche, l'emploi du passé simple crée un écart par rapport à l'actualité présente du narrateur. Nous avons ainsi l'impression que les événements repérés au passé simple sont complètement découpés de l'actualité du narrateur. La combinaison entre le présent et le passé simple crée finalement la relation étroite entre l'énonciation historique et discursive.

\section{Références bibliographiques}

Barceló, Gérard Joan \& Jacques Bres. Les temps de l'indicatif en français. Paris : Ophrys, 2006.

Benveniste, Émile. Problèmes de linguistique générale, I. Paris : Gallimard, 1966. Fleischman, Suzanne. Tense and narrativity; from Medieval performance to modern fiction. London : Routledge, 1990.

Genette, Gérard. Figures III. Paris : Éditions du Seuil, 1972.

Leeman-Bouix Danielle. Grammaire du verbe français : des formes au sens. Paris : Nathan, 1994.

Marmier Xavier. Les Lettres sur l'Adriatique et le Monténégro. Paris : A. Bertrand, 1854. 
Marmier, Xavier. Journal : 1848-1890, tome premier. Genève : Droz, 1968.

Piroska, Sebe-Madacsy, "Lettres de voyage de Petofi Sandor en 1847. " Roman et récit de voyage. Éds. Philipe Antoine \& Marie-Christine Gomez-Géraud. Paris : Presses de l'Université de Paris-Sorbonne, 2001. 217-223.

Riegel, Martin, Jean-Christophe Pellat \& René Rioul. Grammaire méthodique du français. Paris : Presses universitaires de France, 2013.

\section{IMAGE OF MONTENEGRO AND VIEWPOINT ON NARRATION IN XAVIER MAR- MIER'S LETTERS ON THE ADRIATIC AND MONTENEGRO}

In the present article, we intend to present Letters on the Adriatic and Montenegro by Xavier Marmier, a French traveller and writer who took many trips around the world, including Montenegro in 1852. His journey is described in two volumes, and the second one is mainly devoted to Montenegro (six out of eight chapters). He spent two months in Montenegro and wrote a beautiful testimony of this period, relying on the historical facts, testimonies of the inhabitants and on his personal impressions. In the first part of our paper, we provide a summary of his writing on Montenegro, focusing on points such as the historical context, the living conditions of the people, as well as on their customs and traditions. In the second part, we propose a reflection on the narration and more precisely on the narrative temporality in this work. We seek to identify the different temporal markers in order to explain the use of certain tenses in the narrative. porality.

Key words: Montenegro, Xavier Marmier, travel writing, narration, tem- 